\title{
Decreased Motor Function Is Associated with Poorer Cognitive Function in Elderly with Type 2 Diabetes
}

\author{
Elizabeth Guerrero-Berroa ${ }^{a} \quad$ Ramit Ravona-Springer ${ }^{c}$ \\ Anthony Heymann ${ }^{d, e}$ James Schmeidler ${ }^{a}$ Jeremy M. Silverman ${ }^{a}$ b \\ Mary Sano ${ }^{a}$ b Keren Koifmann ${ }^{c}$ Rachel Preiss ${ }^{d}$ Hadas Hoffman $^{d}$ \\ Michal Schnaider Beeri ${ }^{a}, c$ \\ a Department of Psychiatry, Icahn School of Medicine at Mount Sinai, New York, N.Y., and \\ b James J. Peters Veterans Affairs Medical Center, Bronx, N.Y., USA; ${ }^{\mathrm{c} T h e}$ Joseph Sagol \\ Neuroscience Center, Sheba Medical Center, Ramat Gan, dMaccabi Healthcare Services, and \\ e Sackler Faculty of Medicine, University of Tel Aviv, Tel-Aviv, Israel
}

Key Words

Type 2 diabetes · Motor function · Cognition · Elderly · Memory · Dementia

\begin{abstract}
Background/Aims: Impaired motor function has been associated with cognitive impairment and dementia, but this relationship is poorly understood in elderly with type 2 diabetes (T2D). We thus investigated it in a large sample $(n=726)$ of cognitively normal elderly with T2D. Methods: In this cross-sectional study, hierarchical linear regressions assessed correlations of 3 motor measures (timed walk, grip strength, and self-reported motor difficulties) with episodic memory, attention/working memory, semantic categorization, executive function, and overall cognition controlling for demographics. Results: Longer timed walk and weaker grip strength were associated with poorer performance in all cognitive domains except episodic memory. Conclusions: Associations of motor and cognitive functions in T2D and non-T2D samples are consistent. A lack of association of motor function with episodic memory may suggest non-Alzheimer's disease-related underlying mechanisms.
\end{abstract}


Function in Elderly with Type 2 Diabetes

\section{Introduction}

Type 2 diabetes (T2D) is associated with cognitive decline, mild cognitive impairment (MCI) [1], and dementia [2, 3], including Alzheimer's disease (AD) [4]. While memory loss is the hallmark symptom of $\mathrm{AD}$ [5], other cognitive domains (e.g. language) are impaired [6]. Some types of dementia or disease-related cognitive decline are characterized primarily by deficits on other cognitive domains. For example, diminished attention and executive function are observed in both vascular dementia [7] and T2D [8]. Decline in executive function has been linked to functional impairment $[9,10]$ in the elderly. Given the projected increase in the growth of the elderly population by 2050 [11], and in the prevalence of T2D by 2030 [12], its deleterious effects on cognition are a great public health concern.

Thus, identifying risk factors that could identify, prevent, or delay T2D-related cognitive deficits is important. Ideally, these risk factors should be cost-effective and good predictors of cognitive function. Measures of motor function may be one such predictor. In the general population, the association between motor function and cognitive function is well established [13-16]. Among nondemented older adults, measures of motor function differentiated cognitively normal and mildly impaired individuals [17]. Impaired gait (e.g. reduced timed walk/ speed of walking) and diminished muscle strength (e.g. reduced grip strength) have been associated with subsequent dementia and greater rate of cognitive decline $[13,14,16,18-20]$. There is evidence suggesting that decreased muscle strength precedes cognitive deficits - low grip strength was associated with significantly increased risk of both MCI [14] and AD [13, 14]. Impaired motor ability has been found in studies of T2D [21, 22], but reports on the association between cognitive function and motor function in this population are rare.

Although in the general population, the effects of gait abnormalities and muscle strength on cognitive function and progression to dementia have been examined [14-16, 20,23], their associations with cognition in T2D are lacking, especially in a single study and across different cognitive domains. Since both motor and cognitive abilities are affected in T2D, it is possible that there is a common biological mechanism such as brain atrophy [21] and inflammatory responses $[24,25]$ that underlies this association. The goal of this study was to examine the relationships of measures of motor function - timed walk, grip strength, and a self-reported measure on the difficulty performing gross and fine motor activities - with cognitive performance in nondemented T2D elderly. This study builds on the Israel Diabetes and Cognitive Decline (IDCD) study [26], an investigation of the effects of long-term T2D-related characteristics (e.g. inflammation, glycemic control, and obesity) on cognitive decline in T2D elderly.

\section{Materials and Methods}

\section{Participants}

The IDCD randomly recruited community-dwelling T2D elderly (65+ years old) living in central Israel, from approximately 11,000 clients enrolled in the diabetes registry of the Maccabi Healthcare Services (MHS). MHS is the second largest HMO in Israel, treating a representative cross-section of 2 million citizens. The MHS diabetes registry was established in 1998 to facilitate diabetes management and to improve treatment. Any of the following criteria are necessary for enrollment into the registry: (1) hemoglobin A1c (HbA1c) >7.25\%; (2) glucose $>200 \mathrm{mg} / \mathrm{dl}$ on two exams more than 3 months apart; (3) purchase of diabetic medication twice within 3 months supported by an $\mathrm{HbA} 1 \mathrm{c}$ level $>6.5 \%$ or a glucose level $>125$ $\mathrm{mg} / \mathrm{dl}$ within half a year; (4) diagnosis of T2D (ICD-9 code) by a general practitioner, internist, endocrinologist, ophthalmologist, or T2D advisor, supported by an HbA1c level $>6.5 \%$ or a glucose level $>125 \mathrm{mg} / \mathrm{dl}$ within half a year. These criteria have been validated by 20 physi- 
cians in the MHS against their own practice records [27]. The IDCD study design has been previously described [26]. In brief: the baseline inclusion criteria were having T2D; normal cognition at entry; being free of any neurological (e.g. Parkinson's disease, stroke), psychiatric (e.g. schizophrenia) or other diseases (e.g. alcohol or drug abuse) that might affect cognition, and having an informant. Participants were assessed by a physician experienced in the assessment and diagnosis of dementia and by a neuropsychologist, who administered the broad neuropsychological battery. Intact cognition was determined by a diagnostic consensus conference that included neurologists, psychiatrists, and neuropsychologists experienced with dementia, with at least two specialties present. The Clinical Dementia Rating (CDR) scale and neurological and psychiatric assessments were used to ensure intact cognition (CDR score $=0$ ) at study entry. Cognitively impaired participants (CDR score $>0$, including MCI) were excluded from the study and referred back to their primary physician.

The IDCD has recruited 1,288 participants since March 2010, of whom 109 refused to continue; 282 were excluded, primarily due to cognitive impairment (86\%), Parkinson's disease (2\%), stroke (1\%), lack of informant (8\%), or other reasons (3\%); 171 had incomplete data for this study. Thus, this cross-sectional study included the 726 eligible participants with complete data on motor measures, cognitive domains, and covariates (demographics, cardiovascular risk factors, T2D characteristics, and inflammatory markers). The study was approved by the Icahn School of Medicine at Mount Sinai, Sheba Medical Center, and MHS institutional review board committees.

\section{Motor Function Assessment}

Motor function was assessed using two performance-based (timed walk and grip strength) and one self-reported (gross and fine motor difficulties) measures of motor ability, as follows. (1) Timed walk: for this task, participants were instructed to walk $3 \mathrm{~m}$ at their usual pace. The time (in seconds) required to walk $3 \mathrm{~m}$ was recorded. (2) Grip strength: a hydraulic hand dynamometer (Jamar; Lafayette Instrument Co USA), which displays isometric grip force ranging from 0 to $90 \mathrm{~kg}$, was used to measure grip strength bilaterally. Three trials of grip strength from the dominant hand were averaged for a composite grip strength score [28]. (3) Gross and fine motor difficulties: this questionnaire asks questions regarding difficulties performing gross and fine motor activities: 'How difficult is it for you to (a) bend, (b) lift $5 \mathrm{~kg}$, (c) extend arms, (d) write, (e) walk $400 \mathrm{~m}$, and (f) perform house chores?' Each question is rated as 1 (no difficulties) through 5 (very difficult) [28].

\section{Neuropsychological Function Assessment}

Cognitive function at entry was assessed using 12 neuropsychological tests, grouped into cognitive domains according to the factor with the highest loading: (1) episodic memory: Word List Memory, Word List Recall, and Word List Recognition from the Consortium to Establish a Registry for Alzheimer's Disease (CERAD) neuropsychological battery [29, 30]; (2) attention/working memory: Shape Cancellation and Digit Span (forward and backward) from the Wechsler Memory Scale-Revised [31]; (3) language/semantic categorization: Similarities [32], Letter Fluency [33], and Animal Fluency [34], and (4) executive function: Trail Making Test (Parts A and B) [35], CERAD-Constructional Praxis, and Digit Symbol from the Wechsler Adult Intelligence Scale-Revised [32]. Trails A and Shape Cancellation had a kurtosis above 5.0, indicating a distribution with one of more outliers substantially different from a normal distribution - but none of the other neuropsychological tests had a kurtosis above 1.5. These two tests were logarithmically transformed, and Trails A, Trails B and Shape Cancellation were reversed so that a large value represented good cognition. Raw scores were converted to z scores using participants' means and SDs. A composite measure of global cognitive function (overall cognition) was created by averaging all the z scores. Scores for the 
four cognitive domains were calculated as averages of $\mathrm{z}$ scores, with at least two of the $\mathrm{z}$ scores required.

\section{Statistical Analyses}

In hierarchical linear regression analyses for each cognitive domain and overall cognition, three demographic covariates (age, education, and gender) were entered in the first step, followed by three measures of motor function (timed walk, grip strength, and gross and fine motor difficulties). From these analyses we obtained partial simple correlations and partial multiple correlations showing the association of motor function with cognitive function, controlling for demographics. The multiple correlations summarize associations of all three motor function measures with each cognitive measure. Supplemental regression analyses entered three additional sets of covariates, cardiovascular risk factors (body mass index, total cholesterol, and hypertension), T2D characteristics (HbA1c mean, retinopathy, duration of T2D estimated by length of time in the MHS diabetes registry, and whether medication was taken for T2D), and inflammatory markers [interleukin-6 (IL-6) and C-reactive protein]. The cardiovascular risk factors as well as $\mathrm{HbA1c}$ were calculated as the means of all assessments for each participant in the MHS diabetes registry. Other regression analyses included a measure of the extent of depressive symptoms, the score on the 15-item Geriatric Depression Scale [36], as a covariate. With the exception of cardiovascular risk factors and T2D characteristics, which were retrieved from the MHS diabetes registry, all covariates were first measured at the baseline of the IDCD. These variables are potential confounders that are available in the IDCD and that can affect either motor [24, 37] or cognitive function [25, 26, $38]$, and thus may explain the relationship between motor function and cognition.

We note that a motoric component was involved in the performance of several of these tests - all four tests of executive functions, and one of the two attention/working memory tests, Shape Cancellation. For the other tests in the battery, there was no motor component (Digit Span in attention/working memory, and all of the episodic memory and language/ semantic categorization tests).

\section{Results}

\section{Demographic, Clinical, and Motor Function Characteristics}

Table 1 presents the demographic, clinical, and motor function characteristics of the sample, as well as their associations with the three measures of motor function, controlling for demographics. Mean age for the total sample was 71.8 years ( $S D=4.6$, range $=65-84$ ) and mean education was 13.1 years $(\mathrm{SD}=3.4$, range $=0-24,24.4 \%$ with less than 12 years of education). There were more men (61.7\%) than women. The Mini-Mental State Examination mean score was 28.0 ( $\mathrm{SD}=1.7$ ), which is consistent with normal cognition. Being male and younger age were associated with stronger grip strength, and more education was associated with shorter timed walk. Of the T2D characteristics, HbA1c mean was associated with weaker grip strength. Of the three measures of motor function, timed walk was associated with both grip strength and gross and fine motor difficulties, which were not associated with each other.

Hierarchical linear regression analyses, controlling for age, education, and gender, showed that motor function was generally associated with cognitive function (table 2). Episodic memory was the only cognitive domain that was not associated with any of the three measures of motor function; the association between language/semantic categorization and report of gross and fine motor difficulties was also nonsignificant. Timed walk had consistently the strongest association with cognition, followed by grip strength, and then gross and fine motor difficulties. The longer it took to walk $3 \mathrm{~m}$ and the weaker the grip strength, the 
Guerrero-Berroa et al.: Decreased Motor Function Is Associated with Poorer Cognitive Function in Elderly with Type 2 Diabetes

Table 1. Demographic, clinical, and motor function characteristics of the participants $(n=726)$

\begin{tabular}{lcccc}
\hline & Mean \pm SD & \multicolumn{2}{l}{ Partial correlations } & \\
\cline { 3 - 4 } & & timed walk & grip strength & $\begin{array}{c}\text { gross and fine } \\
\text { motor difficulties }\end{array}$ \\
\hline Age, years & & & \\
Male, \% & $71.8 \pm 4.6$ & $0.123^{* *}$ & $-0.227^{* * *}$ & 0.065 \\
Education, years & 61.7 & $-0.084^{*}$ & $0.660^{* * *}$ & $-0.092^{*}$ \\
Body mass index & $13.1 \pm 3.4$ & $-0.217^{* * *}$ & $0.085^{*}$ & -0.028 \\
Total cholesterol, mg/dl & $28.2 \pm 4.3$ & $0.092^{*}$ & -0.015 & $0.100^{* *}$ \\
Hypertension, \% & $180.3 \pm 24.9$ & -0.024 & 0.020 & -0.028 \\
HbA1c, \% & 86.0 & 0.050 & -0.012 & 0.037 \\
& $6.8 \pm 0.77$ & 0.061 & $-0.118^{* *}$ & 0.028 \\
Duration of T2D, years & $10.5 \pm 0.77$ & -0.005 & & 0.025 \\
Retinopathy, \% & 41.3 & 0.006 & -0.047 & -0.003 \\
IL-6 (n = 698), pg/ml & $2.9 \pm 2.6$ & 0.046 & 0.022 & 0.016 \\
CRP (n = 697), $\mu$ g/ml & $1.1 \pm 1.7$ & 0.065 & 0.000 & 0.011 \\
Medication for T2D, \% & 87.3 & 0.040 & -0.052 & 0.011 \\
Geriatric Depression Scale & & & & 0.030 \\
score & $2.2 \pm 2.3$ & $0.144^{* *}$ & $-0.135^{* *}$ & $0.146^{* * *}$ \\
MMSE score & $28.0 \pm 1.7$ & $-0.130^{* *}$ & 0.041 & -0.043 \\
Timed walk, s & $4.6 \pm 2.6$ & - & $-0.308^{* * *}$ & $0.178^{* * *}$ \\
Grip strength & $28.5 \pm 9.8$ & $-0.308^{* * *}$ & - & -0.047 \\
Gross and fine motor & $1.5 \pm 0.9$ & $0.178^{* * *}$ & -0.047 & - \\
difficulties, \% & & & & \\
\hline
\end{tabular}

MMSE = Mini-Mental State Examination; duration of T2D = estimated by length of time in the diabetes registry. Partial correlations: controlling for age, gender, and education. ${ }^{*} \mathrm{p}<0.05$; $^{* *} \mathrm{p}<0.01{ }^{* * *} \mathrm{p}<0.0001$.

Table 2. Partial correlations for the association of motor function with cognitive domains

\begin{tabular}{|c|c|c|c|c|}
\hline \multirow[t]{2}{*}{ Cognitive domains } & \multirow[t]{2}{*}{ Timed walk } & \multicolumn{3}{|c|}{ Motor functions } \\
\hline & & grip strength & $\begin{array}{l}\text { gross and fine } \\
\text { motor difficulties }\end{array}$ & multiple $r$ \\
\hline Executive function & $-0.183^{* * *}$ & $0.168^{* * *}$ & $-0.088^{*}$ & $0.225^{* * *}$ \\
\hline Attention/working memory & $-0.140^{* *}$ & $0.126^{* *}$ & $-0.079 *$ & $0.174^{* * *}$ \\
\hline $\begin{array}{l}\text { Language/semantic } \\
\text { categorization }\end{array}$ & $-0.227^{* * *}$ & $0.112^{* *}$ & -0.043 & $0.231^{* * *}$ \\
\hline Episodic memory & 0.016 & 0.049 & -0.007 & 0.059 \\
\hline Overall cognition & $-0.192^{* * *}$ & $0.164^{* * *}$ & $-0.078^{*}$ & $0.226^{* * *}$ \\
\hline
\end{tabular}

Motor functions: controlling for age, gender, and education. ${ }^{*} \mathrm{p}<0.05 ;{ }^{* *} \mathrm{p}<0.01{ }^{* * *} \mathrm{p}<0.0001$.

lower the scores on executive function, attention/working memory, language/semantic categorization, and overall cognition. The more difficult it was to perform gross and fine motor activities, the lower the scores on executive function, attention/working memory, and overall cognition. All results that were significant at $\mathrm{p}<0.0001$ (indicated by ${ }^{* * *}$ ) remained significant after Bonferroni adjustment for multiple comparisons.

Entering 9 additional covariates, 3 cardiovascular risk factors, 4 T2D characteristics, and 2 inflammatory markers did not change the magnitude of any of the partial simple correlations by more than 0.017 and the category of statistical significance never changed. In contrast, 
inclusion of depressive symptoms as a covariate did reduce the magnitude of observed associations. The category of statistical significance was reduced for grip strength [language/ semantic categorization (from partial $r=0.112, p=0.002$ to partial $r=0.091, p=0.015$ ) and overall cognition (from partial $r=0.164, p=0.000009$ to partial $r=0.144, p=0.000102$ )] and for gross and fine motor difficulties [executive function (from partial $r=-0.088, p=0.017$ to partial $r=-0.065, p=0.079$ ), attention/working memory (from partial $r=-0.079, p=0.034$ to partial $r=-0.069, p=0.063$ ), and overall cognition (from partial $r=-0.078, p=0.035$ to partial $r=-0.054, p=0.149$ )].

The intercorrelations among the three measures of motor function, controlling for age, gender, and education, showed that the longer it took to walk $3 \mathrm{~m}$, the lower the grip strength (partial $r=-0.308, p<0.0001$ ), and the longer it took to walk $3 \mathrm{~m}$, the more difficult it was to perform gross and fine motor activities (partial $r=0.178, p<0.0001$ ). Grip strength and difficulty performing gross and fine motor activities were not associated (partial $r=-0.047, p=$ $0.203)$.

Partial multiple correlations using all three measures of motor function, controlling for age, gender, and education, showed an association of motor function with overall cognition and all cognitive domains ( $\mathrm{p}<0.0001$ ), except for episodic memory (table 2 , last column). Compared with using the most strongly correlated single measure, using all three measures of motor function improved prediction for executive function $(p=0.002)$, attention/working memory $(p=0.019)$, and overall cognition $(p=0.005)$, but not for language/semantic categorization $(p=0.463)$ or episodic memory $(p=0.661)$.

\section{Discussion}

Motor function was analyzed on 726 nondemented elderly with T2D participating in the IDCD study. Our findings on the association of gait abnormalities (e.g. timed walk) and muscle strength (e.g. grip strength) with cognition, among nondemented older individuals with T2D, are consistent with other studies of non-T2D elderly $[13,18,19]$. Longer time spent walking $3 \mathrm{~m}$ and lower grip strength were strongly associated with decreased cognitive function in the areas of executive function, attention/working memory, language/semantic categorization, and overall cognition. Episodic memory was the only cognitive domain that was not associated with any of the measures of motor function, which is consistent with other studies of non-T2D elderly. For instance, one study found that non-memory tasks (verbal fluency and psychomotor speed) were more strongly associated than a memory task with speed of walking at baseline and with decreased walking speed over a period of 7 years [19].

A self-reported measure of the difficulty performing gross and fine motor activities was associated with cognitive function, with increasing difficulty performing these activities being associated with lower scores in executive function, attention/working memory, and overall cognition (but not language/semantic categorization and episodic memory). However, these relationships were less strong than for the performance-based measures of motor function. Fitzpatrick [16] also found that performance-based, but not self-reported, measures of physical function were associated with poor cognitive function. Overall, these findings suggest that performance-based measures have higher predictive accuracy of cognitive function than self-reported measures.

One possible explanation for the association between poor motor function and cognitive dysfunction is that they may share a common disease process. Motor function depends on the integrity of motor control systems throughout the brain, which regulate planning and execution of motor activity [39]. Diminished motor strength could be related to a compromised nervous system that leads to general deficits including cognitive impairment [18]. 
Decreased motor/psychomotor function - demonstrated by decreased processing speed - is part of the aging process and linked to biological factors such as white matter changes [40]. It is important to highlight that episodic memory included no test requiring psychomotor/ processing speed, and was the only domain that was not associated with any of the measures of motor function. The language/semantic categorization domain did not incorporate any tests requiring motoric responses, but it included verbal fluency tasks, which require processing speed. Thus, to tease out the contribution of processing speed to the association, future studies should compare speed versus non-speed tasks in all domains.

Decreased motor/psychomotor function is also associated with conditions such as dementia and depression. Depressive symptoms have been associated with cognitive decline/ dementia [38, 41-43], white matter changes in dementia [44], motor dysfunction [37], and frontostriatal-limbic dysfunction [45]. Depressive symptoms [46] and T2D complications (e.g. neuropathy) can affect test performance, particularly those tasks requiring motor and processing speed. Including the Geriatric Depression Scale depression measure as a covariate affected the association between motor function and cognition, suggesting that depressive symptoms may be a confounder to the association.

AD pathology has been found in cortical and subcortical motor areas (e.g. motor cortex and substantia nigra) $[39,47]$. AD pathology has also been associated with poor motor function $[47,48]$. The association of AD pathology in the substantia nigra with poor motor function has been found in both those with and without dementia/AD [47]. However, it is worth mentioning that AD pathology typically affects memory, which was not associated with motor function in this study.

It is noteworthy that controlling for T2D characteristics and inflammatory markers did not affect the results of the association of motor function with cognition. Results also remained essentially unchanged after controlling for several cardiovascular risk factors. The latter is consistent with findings from other studies that controlled for possible confounders including vascular disease and vascular risk factors, and found that the significant association between motor function and cognitive decline also remained unchanged $[13,14]$, suggesting that cardiovascular risk factors do not confound the association of motor function with cognition. However, this study excluded participants with cerebrovascular disease - which occurs with frequency in T2D [49] - so the effects of cerebrovascular disease on the relationship cannot be ruled out.

Other possible pathologic processes may contribute to the association between motor function and cognition such as inflammation. High serum levels of IL- 6 at baseline were associated with slow gait speed cross-sectionally and with decline in gait speed at follow-up [24]. Inflammatory markers have also been associated with cognitive decline and dementia [25, 50]. However, in this study, IL-6 was among the covariates that did not change the association of motor function with cognition. Alternative pathological explanations for the association have been suggested. In addition to known pathology, central pathways, such as those involved in brain-derived neurotrophic factor, can be triggered by environmental factors such as reduced physical activity and may be involved in the association between motor and cognitive function [39].

Due to the cross-sectional design of this study, we are unable to ascertain causality. Longitudinal studies have shown mixed results. For instance, Boyle [14] reported an association between poor motor function and cognitive decline among initially cognitively intact elderly. In secondary analyses, they excluded participants who developed AD during the first or second year of follow-up, as well as those who were in the bottom $15 \%$ of cognitive performance at baseline, and those who were in the bottom $15 \%$ of muscle function at baseline, and consistently found an association between poor muscle strength and development of AD. However, this association was not found in a study that used a composite measure of physical 
Guerrero-Berroa et al.: Decreased Motor Function Is Associated with Poorer Cognitive Function in Elderly with Type 2 Diabetes

performance (measures of balance, gait, strength, and dexterity) [51]. Yet another study found that cognitive function can also predict motor function [19].

Since the goal of the IDCD study is to investigate the associations of T2D characteristics with cognitive decline/dementia, it did not include a nondiabetic control group. Such a group would have been helpful in determining differences associated with diabetes. This study lacked neuroimaging data that could have helped explain the contribution of brain abnormalities such as white matter lesions to the association of motor and cognitive function. Another limitation of this study is that it lacked neuropathy data. Finally, the selection of motor measures was limited.

Some of the strengths of this study include the large sample size $(n=726)$, a well-characterized diagnosis of T2D and related characteristics, inclusion of performance-based and self-reported measures of motor function, as well as a comprehensive neuropsychological battery that allowed for the examination of specific cognitive domains.

Assessment of motor function has practical implications; it can aid in the identification of elderly who are at risk of cognitive decline/dementia, and be used to indicate therapeutic intervention such as exercise programs $[52,53]$. Because assessment of motor function does not rely on educational attainment and cultural background, its utility in the prediction of cognitive function is particularly important among individuals with low educational attainment and minority elderly.

\section{Acknowledgements}

This study was supported by NIA grants R01 AG034087 to Dr. Beeri and P50 AG005138 to Dr. Sano, the Helen Bader Foundation and the Irma T. Hirschl Scholar award to Dr. Beeri, the American Federation for Aging Research (AFAR) Young Investigator award, and the Alzheimer's Association grant NIRG-11-205083 to Dr. Ravona-Springer.

\section{Disclosure Statement}

The authors have no conflict of interest to declare.

\section{References}

1 Luchsinger JA: Relation of diabetes to mild cognitive impairment. Arch Neurol 2007;64:570-575.

-2 Luchsinger JA: Diabetes mellitus and risk of Alzheimer's disease and dementia with stroke in a multiethnic cohort. Am J Epidemiol 2001;154:635-641.

-3 Schnaider Beeri M, Goldbourt U, Silverman JM, Noy S, Schmeidler J, Ravona-Springer R, Sverdlick A, Davidson M: Diabetes mellitus in midlife and the risk of dementia three decades later. Neurology 2004;63:1902-1907.

4 Ahtiluoto S: Diabetes, Alzheimer disease, and vascular dementia: a population-based neuropathologic study. Neurology 2010;75:1195-1202.

5 Bondi MW: Neuropsychological function and apolipoprotein E genotype in the preclinical detection of Alzheimer's disease. Psychol Aging 1999;14:295-303.

6 Salmon DP: Alzheimer's disease can be accurately diagnosed in very mildly impaired individuals. Neurology 2002;59:1022-1028.

7 Graham NL, Emery T, Hodges JR: Distinctive cognitive profiles in Alzheimer's disease and subcortical vascular dementia. J Neurol Neurosurg Psychiatry 2004;75:61-71.

-8 Nandipati S, Luo X, Schimming C, Grossman HT, Sano M: Cognition in non-demented diabetic older adults. Curr Aging Sci 2012;5:131-135.

9 Martyr A: Executive function and activities of daily living in Alzheimer's disease: a correlational meta-analysis. Dement Geriatr Cogn Disord 2012;33:189-203. 
10 Marshall GA: Executive function and instrumental activities of daily living in mild cognitive impairment and Alzheimer's disease. Alzheimers Dement 2011;7:300-308.

11 US Census Bureau: Current Population Reports. Population Projections of the United States by Age, Race, and Hispanic origin: 1995-2050. Washington, US Census Bureau, 2000.

12 Wild S: Global prevalence of diabetes: estimates for the year 2000 and projections for 2030. Diabetes Care 2004;27:1047-1053.

13 Buchman AS, Wilson RS, Boyle PA, Bienias JL, Bennett DA: Grip strength and the risk of incident Alzheimer's disease. Neuroepidemiology 2007;29:66-73.

14 Boyle PA: Association of muscle strength with the risk of Alzheimer disease and the rate of cognitive decline in community-dwelling older persons. Arch Neurol 2009;66:1339-1344.

15 Alfaro-Acha A: Does 8-foot walk time predict cognitive decline in older Mexicans Americans? J Am Geriatr Soc 2007;55:245-251.

-16 Fitzpatrick AL: Associations of gait speed and other measures of physical function with cognition in a healthy cohort of elderly persons. J Gerontol A Biol Sci Med Sci 2007;62:1244-1251.

17 Kluger A: Clinical features of MCI: motor changes. Int Psychogeriatr 2008;20:32-39.

18 Alfaro-Acha A, Snih SA, Raji MA, Kuo Y-F, Markides KS, Ottenbacher KJ: Handgrip strength and cognitive decline in older Mexican Americans. J Gerontol A Biol Sci Med Sci 2006;61:859-865.

19 Soumaré A: A cross-sectional and longitudinal study of the relationship between walking speed and cognitive function in community-dwelling elderly people. J Gerontol A Biol Sci Med Sci 2009;64:1058-1065.

-20 Waite LM, Grayson DA, Piguet 0, Creasey H, Bennett HP, Broe GA: Gait slowing as a predictor of incident dementia: 6-year longitudinal data from the Sydney Older Persons Study. J Neurol Sci 2005;229-230:89-93.

-21 Kumar R: Association of type 2 diabetes with depression, brain atrophy, and reduced fine motor speed in a 60- to 64-year-old community sample. Am J Geriatr Psychiatry 2008;16:989-998.

$\checkmark 22$ Espeland MA, Miller ME, Goveas JS, Hogan PE, Coker LH, Williamson J, Naughton M, Resnick SM: Cognitive function and fine motor speed in older women with diabetes mellitus: results from the women's health initiative study of cognitive aging. J Womens Health 2011;20:1435-1443.

23 Rosano C: Association between physical and cognitive function in healthy elderly: the health, aging and body composition study. Neuroepidemiology 2005;24:8-14.

-24 Verghese J, Holtzer R, Oh-Park M, Derby CA, Lipton RB, Wang C: Inflammatory markers and gait speed decline in older adults. J Gerontol A Biol Sci Med Sci 2011;66A:1083-1089.

25 Economos A, Wright CB, Moon YP, Rundek T, Rabbani L, Paik MC, Sacco RL, Elkind MSV: Interleukin 6 plasma concentration associates with cognitive decline: the northern Manhattan study. Neuroepidemiology 2013;40: 253-259.

-26 Ravona-Springer R: Haptoglobin 1-1 genotype is associated with poorer cognitive functioning in the elderly with type 2 diabetes. Diabetes Care 2013;36:3139-3145.

27 Heymann AD, Chodick G, Halkin H, Karasik A, Shalev V, Shemer J, Kokia E: The implementation of managed care for diabetes using medical informatics in a large preferred provider organization. Diabetes Res Clin Pract 2006;71:290-298.

28 Fried LP, Tangen CM, Walston J, Newman AB, Hirsch C, Gottdiener J, Seeman T, Tracy R, Kop WJ, Burke G, McBurnie MA: Frailty in older adults: evidence for a phenotype. J Gerontol A Biol Sci Med Sci 2001;56:M146M156.

29 Beeri MS, Schmeidler J, Sano M, Wang J, Lally R, Grossman H, Silverman JM: Age, gender, and education norms on the CERAD neuropsychological battery in the oldest old. Neurology 2006;67:1006-1010.

-30 Welsh KA, Butters N, Mohs RC, Beekly D, Edland S, Fillenbaum G, Heyman A: The Consortium to Establish a Registry for Alzheimer's Disease (CERAD). 5. A normative study of the neuropsychological battery. Neurology 1994;44:609-614.

31 Wechsler D: Wechsler Memory Scale-Revised Manual. San Antonio, Psychological Corporation, 1987.

-32 Godeau P, Frances-Michel C, Wechsler J, Weinbreck P, Wechsler B, Herreman G, Pinaudeau Y: Immunofluorescence study of skin biopsies of healthy skin in bacterial endocarditis. Rev Med Interne 1981;2:29-32.

33 Spreen O, Benton AL: Neurosensory Center Comprehensive Examination for Aphasia (NCCEA), 1977 Revision: Manual of Instructions. Victoria, Neuropsychology Laboratory, University of Victoria, 1977.

34 Newcombe F: Missile Wounds of the Brain: A Study of Psychological Deficits. Oxford, Oxford University Press, 1969.

-35 Reitan RM: Validity of the trail making test as an indicator of organic brain damage. Percept Mot Skills 1958; 8:271-276.

-36 Sheikh JI, Yesavage JA: Geriatric Depression Scale (GDS): recent evidence and development of a shorter version. Clin Gerontol 1986;5:165-172.

-37 Demakakos P, Cooper R, Hamer M, de Oliveira C, Hardy R, Breeze E: The bidirectional association between depressive symptoms and gait speed: evidence from the English Longitudinal Study of Ageing (ELSA). PLoS One 2013;8:e68632.

-38 Wilson RS, Barnes LL, Mendes de Leon CF, Aggarwal NT, Schneider JS, Bach J, Pilat J, Beckett LA, Arnold SE, Evans DA, Bennett DA: Depressive symptoms, cognitive decline, and risk of AD in older persons. Neurology 2002;59:364-370.

39 Buchman AS, Bennett DA: Loss of motor function in preclinical Alzheimer's disease. Expert Rev Neurother 2011;11:665-676. 
40 Salthouse TA: Interrelations of age, visual acuity, and cognitive functioning. J Gerontol B Psychol Sci Soc Sci 1996;51:P317-P330.

-41 Wilson RS, Mendes De Leon CF, Bennett DA, Bienias JL, Evans DA: Depressive symptoms and cognitive decline in a community population of older persons. J Neurol Neurosurg Psychiatry 2004;75:126-129.

-42 Devanand DP, Sano M, Tang MX, Taylor S, Gurland BJ, Wilder D, Stern Y, Mayeux R: Depressed mood and the incidence of Alzheimer's disease in the elderly living in the community. Arch Gen Psychiatry 1996;53:175182.

43 Paterniti S, Verdier-Taillefer MH, Dufouil C, Alperovitch A: Depressive symptoms and cognitive decline in elderly people. Longitudinal study. Br J Psychiatry 2002;181:406-410.

-44 Jonsson M, Edman A, Lind K, Rolstad S, Sjogren M, Wallin A: Apathy is a prominent neuropsychiatric feature of radiological white-matter changes in patients with dementia. Int J Geriatr Psychiatry 2010;25:588-595.

45 Alexopoulos GS: Role of executive function in late-life depression. J Clin Psychiatry 2003;64(suppl 14):18-23

46 Alexopoulos GS, Buckwalter K, Olin J, Martinez R, Wainscott C, Krishnan KR: Comorbidity of late life depression: an opportunity for research on mechanisms and treatment. Biol Psychiatry 2002;52:543-558.

47 Schneider JA, Li JL, Li Y, Wilson RS, Kordower JH, Bennett DA: Substantia nigra tangles are related to gait impairment in older persons. Ann Neurol 2006;59:166-173.

48 Buchman AS: Physical frailty in older persons is associated with Alzheimer disease pathology. Neurology 2008;71:499-504.

49 Kameyamai M, Fushmi H, Udaka F: Diabetes mellitus and cerebral vascular disease. Diabetes Res Clin Pract 1994;24(suppl):S205-S208.

-50 Koyama A, O’Brien J, Weuve J, Blacker D, Metti AL, Yaffe K: The role of peripheral inflammatory markers in dementia and Alzheimer's disease: a meta-analysis. J Gerontol A Biol Sci Med Sci 2013;68:433-440.

51 Albert MS: Predictors of cognitive change in older persons: MacArthur studies of successful aging. Psychol Aging 1995;10:578-589.

52 Liu-Ambrose T, Nagamatsu LS, Graf P, Beattie BL, Ashe MC, Handy TC: Resistance training and executive functions: a 12-month randomized controlled trial. Arch Intern Med 2010;170:170-178.

53 Larson EB: Physical activity for older adults at risk for Alzheimer disease. JAMA 2008;300:1077-1079. 